\title{
RELATIVISTIC POSITRON-ELECTRON-ION SHEAR FLOWS AND APPLICATION TO GAMMA-RAY BURSTS
}

\author{
Edison Liang ${ }^{1}$, Wen Fu ${ }^{1}$, Markus Boettcher ${ }^{2,3}$, IAn SMith ${ }^{1}$, And Parisa Roustazadeh ${ }^{1,3}$ \\ ${ }^{1}$ Rice University, Houston, TX 77005, USA; liang@ rice.edu \\ ${ }^{2}$ Centre for Space Research, North-West University, Potchefstroom 2520, South Africa \\ ${ }^{3}$ Ohio University, Athens, OH 45701, USA \\ Received 2013 September 13; accepted 2013 November 21; published 2013 December 6
}

\begin{abstract}
We present particle-in-cell simulation results of relativistic shear flows for hybrid positron-electron-ion plasmas and compare to those for pure $e^{+} e^{-}$and pure $e^{-}$ion plasmas. Among the three types of relativistic shear flows, we find that only hybrid shear flow is able to energize the electrons to form a high-energy spectral peak plus a hard power law tail. Such electron spectra are needed to model the observational properties of gamma-ray bursts.
\end{abstract}

Key words: gamma-ray burst: general - magnetic fields - plasmas - relativistic processes

Online-only material: color figures

\section{INTRODUCTION}

Recent large-scale particle-in-cell (PIC; Birdsall \& Langdon 1991) simulations of relativistic shear boundary layers (SBLs) demonstrated efficient generation of ordered magnetic fields and particle energization from initially unmagnetized plasmas (Alves et al. 2012; Liang et al. 2013; Grismayer et al. 2013). In addition to blazar jets (Boettcher 2007; Ghisellini et al. 2005), relativistic SBLs are likely to occur in gamma-ray burst (GRB) jets, which involve ultra-relativistic outflows from stellar-mass collapsed objects interacting with stationary circumstellar and interstellar media (Piran 2000, 2005; Meszaros 2002). Previous PIC simulations of SBLs focused on pure leptonic $\left(e^{+} e^{-}\right.$pairs; Liang et al. 2013) or pure $e^{-}$ion flows with low bulk Lorentz factors (Alves et al. 2012; Grismayer et al. 2013). Here we generalize the previous works to include hybrid $e^{+} e^{-}$ion plasmas at high Lorentz factors $\left(p_{\mathrm{o}}=15\right.$, where $p_{\mathrm{o}}$ is the initial bulk flow Lorentz factor in the center of momentum (CM) frame). We find that only hybrid SBL can accelerate electrons to form a broad high-energy spectral peak followed by a hard power law tail, resembling the typical observed GRB spectrum (Preece et al. 2000; Meegan et al. 2009). In this Letter we summarize the PIC simulation results and discuss their potential applications to the optically thin synchrotron (OTS) model of GRB emissions (Piran 2005; Meszaros 2002). Our simulations are performed in the CM frame in contrast to some MHD simulations which use the laboratory frame of the central engine (Mizuno et al. 2007).

We first summarize the results of pure $e^{+} e^{-}$and pure $e^{-}$ion relativistic SBLs. In the pure $e^{+} e^{-}$case (Liang et al. 2013), transverse electromagnetic (EM) fields are first generated by oblique Weibel-like (Weibel 1959; Yoon 2007; Yang et al. 1993, 1994) and two-stream (Boyd \& Sanderson 2003; Lapenta et al. 2007) instabilities driven by interface-crossing counter streams. The small-scale fields then self-organize into larger and larger ordered quasi-periodic magnetic flux ropes and electric field channels, with alternating polarity. Later they coalesce into large dipolar EM "vortices" extending over hundreds of skin depths $\left(=c / \omega_{\mathrm{e}}, \omega_{\mathrm{e}}=\right.$ electron plasma frequency). The ultimate sizes of these dipolar EM vortices are likely limited by the kinetic energy inflow rate versus turbulence dissipation rate. Particles are energized by cross-field drift motions (Boyd \& Sanderson 2003), leading to a nonthermal soft quasi-power law which turns over at $\sim p_{\mathrm{o}} / 2$. A density trough is formed at the SBL due to plasma expulsion by the extra magnetic pressure. The asymptotic field energy is $\sim 8 \%$ of the total energy (Liang et al. 2013).

For pure $e^{-}$ion $\left(m_{\mathrm{i}} / m_{\mathrm{e}}=1836\right)$ shear flow, the properties of the SBL are drastically different. In the $p_{\mathrm{o}} \leqslant 3$ cases studied by Alves et al. (2012) and Grismayer et al. (2013), the initial instability is a kinetic version of the Kelvin-Helmohltz instability (Chandrasekhar 1981) with free-streaming ions and fluid-like electrons (Gruzinov 2008; Nishikawa et al. 2012). We have extended the pure $e^{-}$ion runs to $p_{\mathrm{o}}=15$ using much larger boxes. As we see below in Section 2, current sheets are formed on opposite sides of the interface, sustaining a steady slab of monopolar magnetic field. The strong magnetic pressure expels the plasma, forming a density trough at the interface, but the ions are expelled more than the electrons due to larger ion gyroradii. This leads to charge separation and formation of a triple layer, with electric fields pointing from both current sheets toward the center of the SBL. Electrons are efficiently accelerated by cross-field drift motions, eventually reaching the ion energy. At late times the electron distribution forms a narrow peak at the decelerated ion energy $\sim p_{\mathrm{o}} m_{\mathrm{i}} c^{2} / 2$ with sharp cutoff: there is no evidence of any power law tail beyond the peak. The asymptotic field energy is $\sim 12 \%$ of the total energy (see Section 2 ).

\section{PIC SIMULATION RESULTS}

We used two PIC codes in this work: the 2.5D (twodimensional (2D) space, 3-momenta) Zohar from LLNL (Birdsall \& Langdon 1991; Langdon \& Lasinski 1976) and the three-dimensional (3D) EPOCH from the University of Worwick, UK, the academic open source version of the code PSC (Ruhl 2006). We compared results from both codes for many different runs and with Alves et al. (2012) and validated their agreement. We also checked that the relativistic Cerenkov and other numerical instabilities (Birdsall \& Langdon 1991) are well suppressed in both codes: total energy is conserved to $<0.1 \%$ in Zohar runs and few percent in EPOCH runs. Figure 1(a) shows the problem setup in the CM frame. 2D runs in the $x-y$ plane are referred to as $P$-mode and $2 \mathrm{D}$ runs in the $y-z$ plane are referred to as $T$-mode (Liang et al. 2013). We have studied both modes, but found that the T-mode instabilities grow very slowly compared to the P-mode for $p_{o}=15$ when ion energy dominates. 3D runs also show that gross SBL properties and the asymptotic electron spectra are similar to 2D $P$-mode 

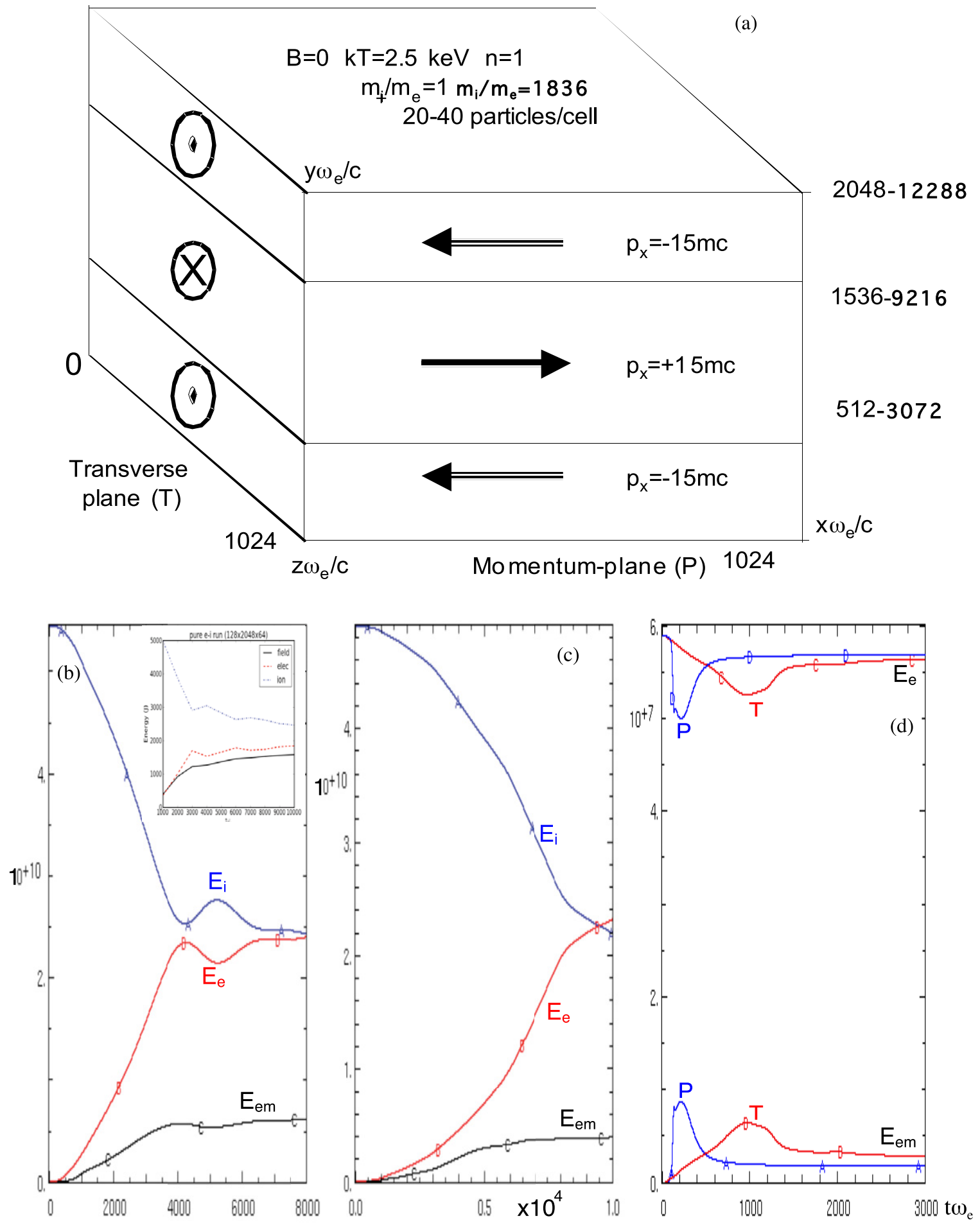

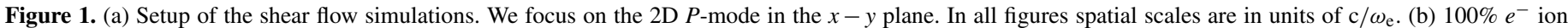

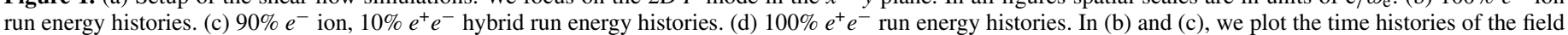

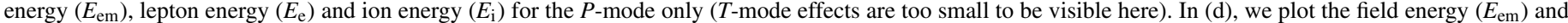
lepton energy $\left(E_{\mathrm{e}}\right)$ for the $P$-mode and $T$-mode separately (from Liang et al. 2013). Panel (b) inset: energy histories for $3 \mathrm{D}$ (128 $\left.\times 2048 \times 64\right) e^{-}$ion run.

(A color version of this figure is available in the online journal.)

(see below). While we will show some 3D results for comparison (Figure 1(b) inset and Figure 4), this Letter will focus on the 2D P-mode results since we can use much larger boxes in 2D. We use doubly periodic $x-y$ boxes ranging from $1024 \times$ 2048 (Zohar) to $1024 \times 12,288$ (EPOCH) with 20-40 particles per cell. The larger EPOCH box allows us to run much longer without interaction between the two shear layers. The initial shear Lorentz factor $p_{\mathrm{o}}=15$ in the $\mathrm{CM}$ frame, much higher than the cases studied by Alves et al. (2012). The plasma is initially unmagnetized. The initial temperature $T=2.5 \mathrm{keV}$ for electrons, positrons and ions $\left(m_{+} / m_{\mathrm{e}}=1, m_{\mathrm{i}} / m_{\mathrm{e}}=1836\right.$, ions carry almost all initial energy). The initial electron density $n$ is normalized to 1 so that cell size = plasma skin depth. We set time step $=0.1 / \omega_{\mathrm{e}}$ so that Boris rotation steps remain stable 
(Birdsall \& Langdon 1991) even in the strongest field regions. Spatial distances are measured in $\mathrm{c} / \omega_{\mathrm{e}}$ and time is measured in $1 / \omega_{\mathrm{e}}$. All physical quantities refer to the CM frame.

We compare the SBLs of three different plasmas: (A) pure $e^{-}$ion, (B) $90 \% e^{-}$ion $+10 \% e^{+} e^{-}$(hybrid case), (C) pure $e^{+} e^{-}$(from Liang et al 2013). Figures 1(b)-(d) compare the time evolution of the different energy components for the three different plasmas. At late times, the field energy saturates at $\sim 12 \%$ of total energy for case (A) and $\sim 8 \%$ for cases (B), and (C) if we sum the $P$-mode and $T$-mode (Liang et al. 2013). These saturation levels appear insensitive to the box size and are much higher than MHD results (Zhang et al. 2009). Figures 1(b) and (c) show that the lepton energy eventually reaches equipartition with the ion energy, but case (B) takes longer than case (A). However the $e^{-}$ion equipartition time scales $\sim$ linearly with $y$-dimension, suggesting that effective $e^{-}$ion coupling via plasma instabilities begins at the SBL but eventually reaches the whole box. Thus relativistic SBLs efficiently convert bulk flow energy into EM fields, which in turn energize the leptons and radiate efficiently. In Figure 1(b) inset shows the energy histories of our largest 3D $(128 \times 2048 \times 64) e^{-}$ion run. Compared to 2D $P$-mode we note that the $3 \mathrm{D}$ field energy is higher (>sum of 2D $P$ and $T$ modes), lepton energy is lower and the leptons take longer to reach equipartition with the ions. Some of these differences are caused by the nonlinear excitation of oblique modes, but some may also be due to the smaller 3D box and numerical effects. The true 3D physical effects can only be settled using much larger runs (e.g., $1024 \times 2048 \times 1024$ ) with higher fidelity, which is beyond the scope of this paper. Figure 2 compares the $\mathbf{B}_{\mathbf{z}}$ and $\mathbf{E}_{\mathbf{y}}$ profiles (the dominant components) for all three plasmas and Figure 3 compares the $\mathbf{J}_{\mathbf{x}}$, net charge $n_{+}-n_{-}$and vertical density profile $n(y)$ (averaged over $x$ ) for cases (A) and (B) at $t \omega_{\mathrm{e}}=3000$. As in Alves et al. (2012), we find that pure $e^{-}$ion shear flows create a monopolar slab of uniform $\mathbf{B}_{\mathbf{z}}$ along the shear interface, sustained by $\mathbf{J}_{\mathbf{x}}$ currents due to different electron and ion drift velocities and densities (Figure 3(c) and (f)), in contrast to the dipolar EM "flywheels" of the pure $e^{+} e^{-}$shear flows (Figure 2(c) and (f); Liang et al 2013). Plasma is expelled from the shear interface by the extra magnetic pressure, creating a density trough (Figure 3(c) and (f)). However, in cases (A) and (B), the leptons, with smaller gyroradii, are less displaced than the ions, leading to charge separation and formation of a triple layer (Figure 3(c) and (f)), with the electric fields pointing from both ion-dominated current sheets toward the electron-dominated center (Figure 2(d) and (e)). The cross $\mathbf{E}, \mathbf{B}$ fields efficiently accelerate the leptons. The hybrid $e^{+} e^{-}$ion SBL is different from both the pure $e^{-}$ ion and pure $e^{+} e^{-}$SBL: outside the hybrid triple layer which is thinner than the pure $e^{-}$ion triple layer, wavy EM fields (Figure 2(b) and (e)) are created which provide additional channels of particle acceleration.

In Figure 4(a) we compare the electron spectra $f(\gamma)(\gamma=$ Lorentz factor) of the three different cases at $t \omega_{\mathrm{e}}=3000$. The pure $e^{+} e^{-}$spectrum peaks at $\gamma \sim p_{\mathrm{o}} / 2$ with a soft highenergy tail produced by stochastic acceleration of vortical EM turbulence (Liang et al. 2013). For pure $e^{-}$ion case (A), the electron spectrum consists of a broad low-energy component plus a narrow high-energy peak at $\gamma \sim p_{\mathrm{o}} m_{\mathrm{i}} / 2 m_{\mathrm{e}}(\sim$ decelerated ion energy at late times) with steep cutoff. The electrons in the high-energy peak are mainly energized by the triple-layer: the steep cutoff is due to the uniformity of the potential gap across the triple layer (Figure 2(d)), and the absence of nonthermal tail above the peak is due to the lack of additional stochastic acceleration by waves. In contrast, for the hybrid $e^{+} e^{-}$ion case (B), the broad low-energy component, which is mainly energized by wave turbulence (see Figure 5), merges with the (weaker) high-energy peak energized by the triple layer to form a quasi-power law. Figure 4(b) shows the detailed electron spectral evolution for the hybrid case. By $t \omega_{\mathrm{e}}=45,000$, not only has the electron peak migrated to the decelerated ion energy $\sim p_{\mathrm{a}} f m_{\mathrm{i}} / 2 m_{\mathrm{e}}(f=$ ion fraction), a well-defined power law tail also formed above the peak with slope $\sim-3$. For OTS and Compton radiation, such electron slope corresponds to a photon index $\sim-2$ (Rybicki \& Lightman 1979). In Figure 4(c) we compare the pure $e^{-}$ion electron spectra in 3D $(128 \times$ $2048 \times 64)$ versus 2D: the 3D peak is broader, but its location and the steep cutoff are identical to the $2 \mathrm{D}$ spectrum. The slight broadening is expected since in $3 \mathrm{D}$ the triple-layer potential gap is less uniform than in $2 \mathrm{D}$ due to oblique mode perturbations. In Figure 4(d) we show the electron phase plot of the hybrid case at $t \omega_{\mathrm{e}}=10,000$ : the momentum distribution is highly anisotropic so that synchrotron radiation would be strongly beamed along $\mathbf{x}$ even in the CM frame. Figure 5 shows the time-lapse $\mathbf{B}_{\mathbf{z}}$ evolution of our largest 2D hybrid run. The slab fields at the SBL stop expanding after $t \omega_{\mathrm{e}} \sim 20,000$, whereas the wavy fields outside the slab continue to expand over time and coalesce into large alternating dipolar flux tubes.

\section{APPLICATION TO GRB}

GRB prompt emission spectra exhibit a broad spectral peak at $E_{\mathrm{pk}} \sim 0.2-0.5 \mathrm{MeV}$ (Preece et al. 2000) plus a power law tail with photon index $\beta \sim-2$ to -2.5 (Band 1993), extending to $>10 \mathrm{GeV}$ in many Fermi bursts (Abdo et al. 2009). In some Fermi LAT bursts there is also evidence of a second hard component (Ackermann et al. 2011; Kocevski et al. 2012). Historically, most GRB models invoke OTS emission by powerlaw electrons (Piran 2005; Meszaros 2002) and interpret $E_{\mathrm{pk}}$ as the synchrotron critical frequency (Rybicki \& Lightman 1979) of the electron peak energy. Recently there was a revival of the Thomson-thick photospheric model (Giannios \& Spruit 2008; Liang 1997; Liang et al. 1997). Both models have their limitations and may apply to different subclasses of GRB spectra: the former may be more relevant to spectra with soft slope below $E_{\mathrm{pk}}$ (Band (1993) $\alpha$ index $>-1$, cf. Preece et al. 2000), while the latter more relevant to spectra with hard slope below $E_{\mathrm{pk}}$ (Band $\alpha<+1$; Preece et al. 2000; Crider et al. 1997). Here we focus on the OTS interpretation of $E_{\mathrm{pk}}$. The Thomsonthick photospheric model will be studied in a later paper. For OTS models, the observed properties of GRBs constrain the emission region parameters (density, magnetic field, size; Piran 2005; Liang \& Noguchi 2009). In addition to the internal shock scenario (Piran 2005; Meszaros 2002), SBL is also a strong candidate since SBL naturally arises when a relativistic jet passes through stationary medium. If we apply the pure $e^{+} e^{-}$ SBL spectrum (Figure 4(a)) to model the OTS $E_{\mathrm{pk}}$ value, we find that it predicts emission parameters orders of magnitudes different from the observed GRB values (too low $E_{\mathrm{pk}}$, see below), which rules out the pure $e^{+} e^{-}$SBL model. If we use the pure $e^{-}$ion SBL electron spectrum to model $E_{\mathrm{pk}}$, it predicts emission parameters consistent with observed GRB values, but it cannot produce any hard power law tail above $E_{\mathrm{pk}}$ (Figure 4(c)), which is observed in all GRBs. This dilemma drove us to explore the hybrid $e^{+} e^{-}$ion SBL model, which is able to produce both high $E_{p k}$ and a hard power law tail above the peak (Figure 4(b)). 

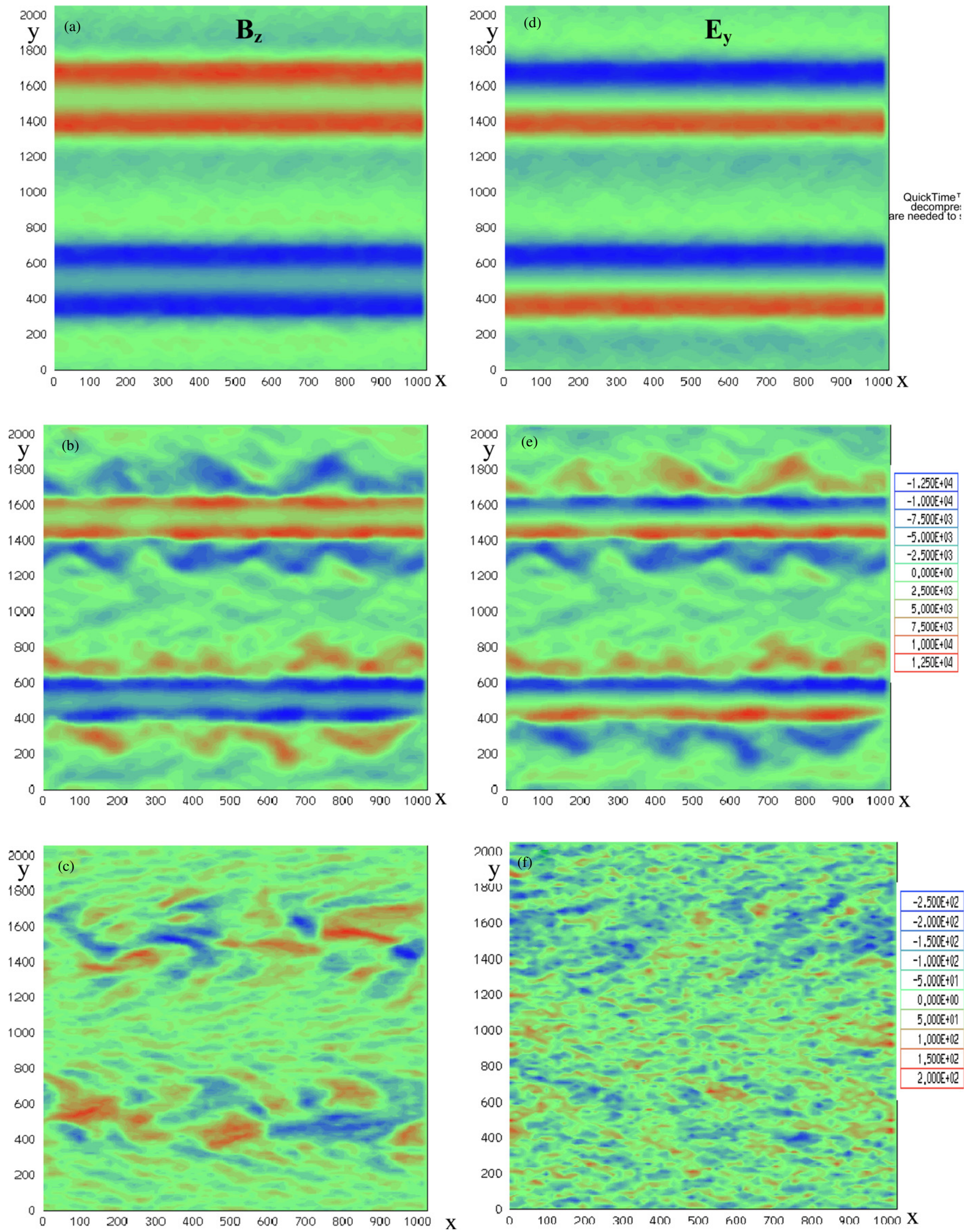

Figure 2. Left: $\mathbf{B}_{\mathbf{Z}}$ (in and out of the plane) contour plots with $p_{\mathrm{o}}=15$ at $t \omega_{\mathrm{e}}=3000$ : (a) $100 \% e^{-}$ion; (b) $90 \% e^{-}$ion, $10 \% e^{+} e^{-}$; (c) $100 \% e^{+} e^{-}$. The transition from a monopolar slab pattern to dipolar vortical "flywheel" pattern is evident. Right: $\mathbf{E}_{\mathbf{y}}$ contour plots for the same three cases showing the transition from a flat triple layer in pure $e^{-}$ion case (d) to triple layer plus EM waves in hybrid case (e) to oblique electric channels in pure $e^{+} e^{-}$case (f) (Liang et al. 2013). Color bar refers to both left and right figures. Units are arbitrary. 

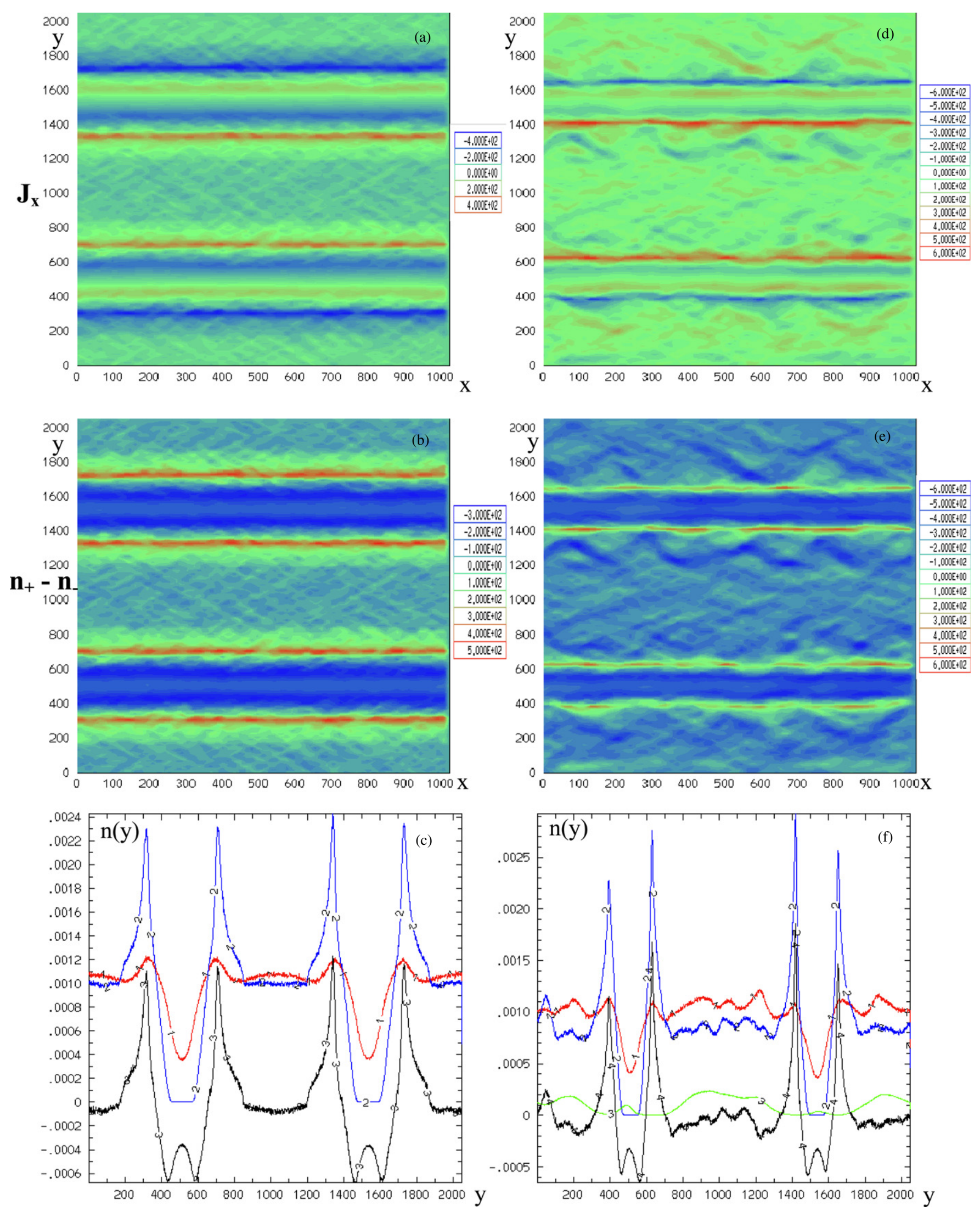

Figure 3. Left: panels (a)-(c) are for pure $e^{-}$ion SBL. Right: panels (d)-(f) are for hybrid $e^{+} e^{-}$ion SBL. All figures refer to $t \omega_{\mathrm{e}}=3000$. Panels (a) and (d) show the current distribution $\mathbf{J}_{\mathbf{x}}$. Stronger outer current sheets are dominated by ions, weaker inner current sheets are dominated by electrons. Panels (b) and (e) show the net charge distribution $\boldsymbol{n}_{+}-\boldsymbol{n}_{-}$. The hybrid triple layer in (e) is thinner than the pure $e^{-}$ion triple layer in (b). Color bar refers to figure to the left. Units are arbitrary. Panels (c) and (f) are the $x$-averaged vertical density profiles $n(y)$. For panel (c), the labels are: 1 electrons, 2 ions, 3 net charge density. Initial densities are normalized to 0.001 . For panel (f), the labels are: 1 electrons, 2 ions, 3 positrons, 4 net charge density. Initial densities are normalized to 0.001 for electrons, 0.0001 for positrons, and 0.009 for ions. In both cases the ions are completely evacuated at the SBL, but the leptons are not, creating net charge separation and formation of the triple layer. The positrons are largely expelled from the ion-dominated current sheets (spikes). 

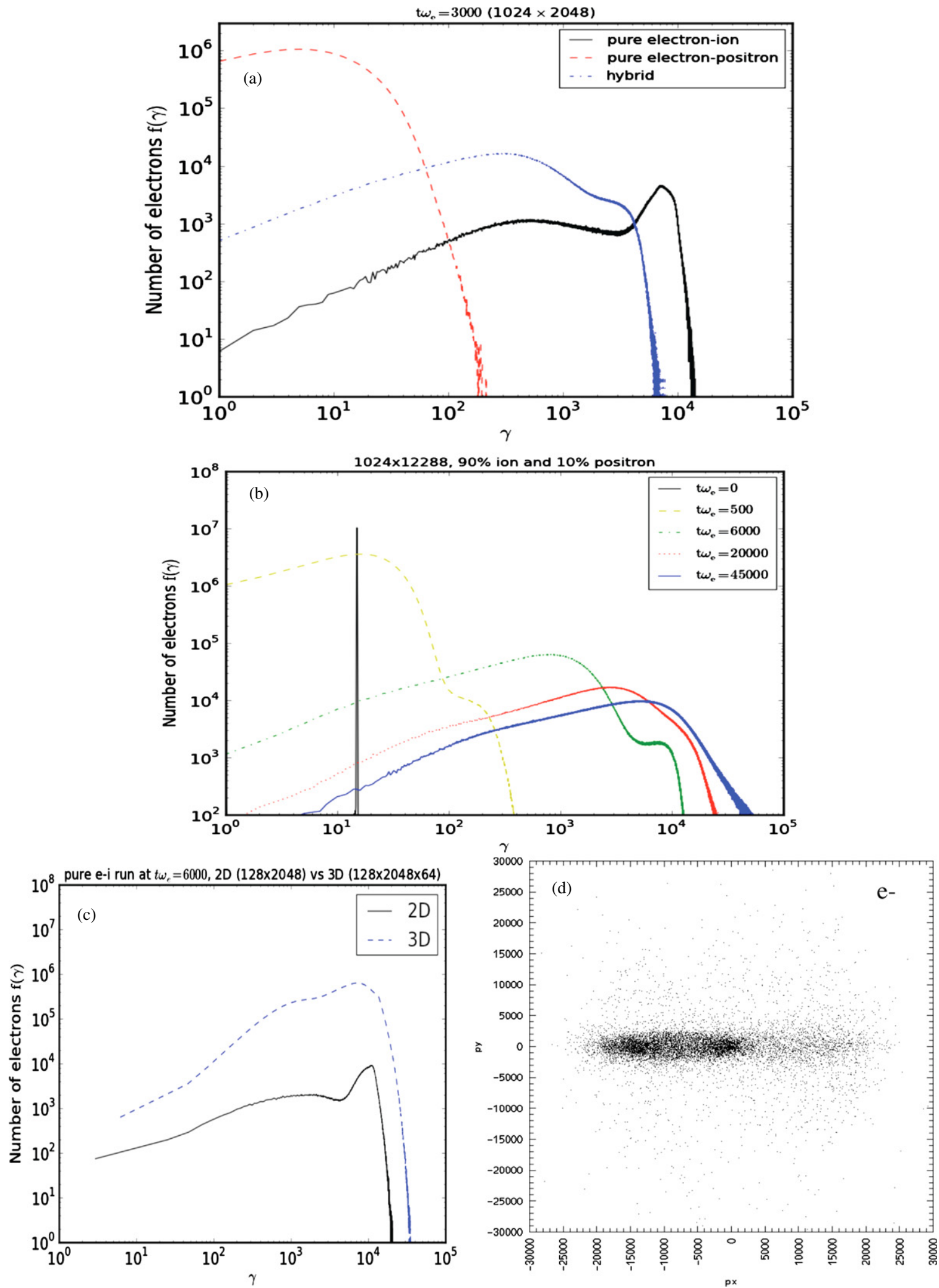

Figure 4. (a) Comparison of the electron spectrum $f(\gamma)$ for the three different plasmas at $t \omega_{\mathrm{e}}=3000$. Spectrum for pure $e^{+} e^{-}$plasma (dashed) has a broad peak at $\sim p_{\mathrm{o}} / 2$ plus a soft nonthermal tail (Liang et al. 2013). Electron spectrum for pure $e^{-}$ion plasma (solid) shows a narrow electron peak at $\sim p_{\mathrm{o}} m_{\mathrm{i}} / 2 m_{\mathrm{e}}$ with steep cutoff. Both spectra show little further evolution for $t \omega_{\mathrm{e}} \gg 3000$. In contrast, electron spectrum for the hybrid case (dot-dashed) exhibits a broad peak with nonthermal high-energy tail, both of which migrate toward higher energy as time progresses. (b) Electron spectral evolution for the hybrid case showing a broad peak at $\sim p_{\mathrm{o}} f m_{\mathrm{i}} / 2 m_{\mathrm{e}} \mathrm{plus}$ hard power law of slope $\sim-3$ above the peak at $t \omega_{\mathrm{e}}=45,000$. (c) Comparison of 3D electron spectrum with 2D at $t \omega_{\mathrm{e}}=6000$ for $e^{-}$ion run (vertical scales not normalized). (d) $p_{\mathrm{x}}$ vs. $p_{\mathrm{y}}$ phase plot for the hybrid case at $t \omega_{\mathrm{e}}=10,000$ for initially left-moving electrons.

(A color version of this figure is available in the online journal.) 


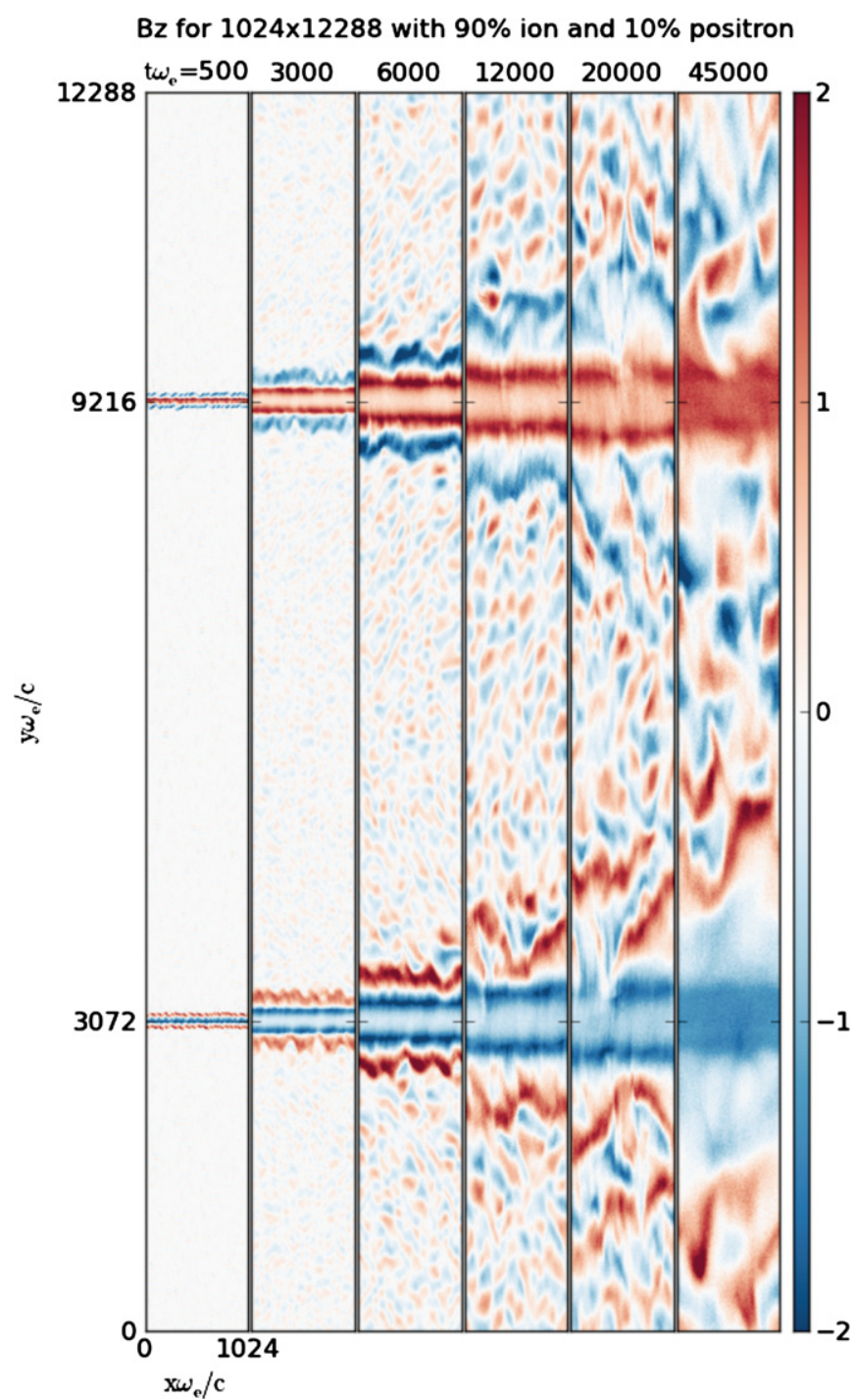

Figure 5. Time-lapse snapshots of $\mathbf{B}_{\mathbf{z}}$ contour plots for the $(1024 \times 12288)$ hybrid $e^{+} e^{-}$ion run (color bar has been renormalized between different panels). The larger $y$-axis allows the problem to run much longer without interaction between the two SBLs.

The hybrid SBL results show that the electron peak energy in the CM frame lies at $\mathrm{e}_{\mathrm{pk}} \sim p_{\mathrm{o}} m_{\mathrm{i}} c^{2} f / 2$, where $f=$ ion fraction, $2 p_{\mathrm{o}}{ }^{2}=\Gamma$ (= bulk Lorentz factor in the host galaxy frame $)$, and the field energy is $\sim 8 \%$ of total energy (Figure 1(c)). Modeling the detailed GRB spectra from PIC simulations involves synthesizing radiation from many particle tracks (Martins et al. 2009; Sironi \& Spitkovsky 2009; Frederiksen et al. 2010; Nishikawa et al. 2011, 2012), which is beyond the scope of this Letter. However, since $\mathbf{B}$ is not too turbulent (Figure 2) and electrons are mainly accelerated orthogonal to field lines, we are justified to use the classical synchrotron formula for a rough orderof-magnitude estimate. The critical synchrotron frequency (Rybicki \& Lightman 1979) observed on Earth, after boosting from the CM frame to the GRB frame with the Lorentz factor $p_{\mathrm{o}}$ and redshifted by $z$ of the host galaxy, is given by $E_{\mathrm{pk}} \sim$ $p_{\mathrm{o}}\left(\mathrm{e}_{\mathrm{pk}} / m_{\mathrm{e}} c^{2}\right)^{2} h v_{c} /(1+z)$, where $v_{c}=e B / 2 \pi m c$ is the electron gyrofrequency in the $\mathrm{CM}$ frame. For example, with $p_{\mathrm{o}}=15$ $(\Gamma=450), f=0.9, z=1$ and $E_{\mathrm{pk}}=250 \mathrm{keV}$ (Preece et al. 2000 ), the above formula predicts $B$ (magnetic field in the CM frame) $\sim 2 \times 10^{4} \mathrm{G}$. Using $B^{2} / 4 \pi p_{\mathrm{o}} n m_{\mathrm{i}} c^{2} \sim 8 \%$, we obtain ion ( electron) density $n$ (in the CM frame) $\sim 10^{10} \mathrm{~cm}^{-3}$. These predicted $(n, B)$ values are consistent with those deduced empirically from GRB observations (Piran 2000, 2005; Liang \& Noguchi 2009). In contrast, if we had used the pure $e^{+} e^{-}$SBL electron peak energy $\mathrm{e}_{\mathrm{pk}} \sim p_{\mathrm{o}} m_{\mathrm{e}} c^{2} / 2$, the predicted $(n, B)$ values would be orders of magnitude off the commonly accepted GRB values. Hence only ion-dominated SBLs can energize electrons to a sufficiently high electron peak energy required by the OTS model. But we also need a finite $e^{+} e^{-}$component to generate additional EM wave turbulence outside the SBL, to stochastically accelerate electrons to form the hard power law above the peak. This result demonstrates the power of kinetic simulations for relativistic plasmas. Another question is the effect of radiation damping on particle acceleration in the current model. For $n \sim 10^{10} \mathrm{~cm}^{-3}, \omega_{\mathrm{e}} \sim 10^{10} \mathrm{rad} \mathrm{s}^{-1}$. Hence $t \omega_{\mathrm{e}}=45,000$ (Figure 4(c)) corresponds to $t \sim 4 \times 10^{-6} \mathrm{~s}$. For $B \sim 10^{4} \mathrm{G}$ and $\gamma_{\max } \sim 5 \times 10^{4}$ (Figure $4(\mathrm{c})$ ), the synchrotron cooling time for electrons is $\sim 2 \times 10^{-4} \mathrm{~s}$, or $\sim 50$ times longer than the acceleration time. Thus we are justified in ignoring radiation damping for the PIC simulations presented here. For much higher $\gamma$ and/or $B$, we may need to include radiation damping effects (Jaroschek \& Hoshino 2009; Noguchi et al. 2005).

Realistic GRB scenarios likely involve hybrid $e^{+} e^{-}$ion jets since the central engine is so hot that pairs should be copiously created (Piran 2005), and the jet likely entrains large amount of baryonic matter while emerging from the collapsar. Observationally, a small pair fraction may be difficult to detect directly. But if the pair fraction is large, Faraday rotation effects on polarized radiation may be detectable since positrons and electrons induce opposite rotations. We have only scratched the tip of the hybrid SBL parameter space. Besides varying $f$ and $p_{\mathrm{o}}$, future simulations will explore the effects of density and composition jumps across the shear interface and embedded primordial magnetic fields in the jet.

This work was supported by NASA Fermi Cycle 4 and 5 grants NNX12AE31G and NNX12AO81G. We thank Professor Arber of the University of Worwick, UK, for providing the EPOCH code. EPOCH runs on Rice clusters were supported by the Data Analysis And Visualization Cyberinfrastructure funded by NSF grant OCI-0959097. ZOHAR runs were performed at Lawrence Livermore National Laboratory.

\section{REFERENCES}

Abdo, A. A., Ackermann, M., Ajello, M., et al. 2009, Natur, 462, 331 Ackermann, M., Ajello, M., Asano, K., et al. 2011, MNRAS, 412, L20 Alves, P., Grismayer, T., Martins, S. F., et al. 2012, ApJL, 746, L14 Band, D. 1993, ApJ, 413, 281

Birdsall, C., \& Langdon, A. B. 1991, Plasma Physics via Computer Simulation (Bristol: IOP)

Boettcher, M. 2007, Ap\&SS, 309, 95

Boyd, T., \& Sanderson, J. 2003, The Physics of Plasmas (Cambridge: Cambridge Univ. Press)

Chandrasekhar, S. 1981, Hydrodynamic and Hydromagnetic Stability (New York: Dover)

Crider, A., Liang, E. P., Smith, I. A., et al. 1997, ApJL, 479, L39

Frederiksen, J., Haugbølle, T., Medvedev, M. V., \& Nordlund, A. 2010, ApJ, 722, L114

Ghisellini, G., Tavecchio, F., \& Chiaberge, M. 2005, A\&A, 432, 401

Giannios, D., \& Spruit, H. C. 2008, A\&A, 469, 1

Grismayer, T., Alves, E. P., Fonseca, R. A., \& Silva, L. O. 2013, PhRvL, 111,015005

Gruzinov, A. 2008, arXiv:0803.1182

Jaroschek, C. H., \& Hoshino, M. 2009, PhRvL, 103, 075002

Kocevski, D., Chiang, J., Connaughton, V., Briggs, M., \& Moretti, E. 2012, ApJ, submitted (arXiv:1201.3948)

Langdon, A. B., \& Lasinski, B. 1976, Meth. Comp. Phys. 16, ed. B. Alder et al. (New York: Academic Press), 327 
Lapenta, G., Markidis, S., Marocchino, A., \& Kaniadakis, G. 2007, ApJ, 666,949

Liang, E. 1997, ApJ, 491, L15

Liang, E., Boettcher, M., \& Smith, I. 2013, ApJ, 766, L19

Liang, E., Kusunose, M., Smith, I. A., \& Crider, A. 1997, ApJ, 479, L35

Liang, E., \& Noguchi, K. 2009, ApJ, 705, 1473

Martins, J. L., Martins, S. F., Fonseca, R. A., \& Silva, L. O. 2009, Proc. SPIE, 7359, 73590V-1

Meegan, C., Lichti, G., \& Bhat, P. N. 2009, ApJ, 702, 791

Meszaros, P. 2002, ARA\&A, 40, 137

Mizuno, Y., Hardee, P., \& Nishikawa, K.-I. 2007, ApJ, 662, 835

Nishikawa, K., Hardee, P., Zhang, B., et al. 2013, AnGeo, 31, 1535

Nishikawa, K., Niemiec, J., \& Medvedev, M. 2011, AdSpR, 47, 1434

Nishikawa, K., Niemiec, J., Zhang, B., et al. 2012, IJMPS, 8, 259
Noguchi, K., Liang, E., \& Nishimura, K. 2005, NCimC, 28, 381

Piran, T. 2000, PhR, 333, 529

Piran, T. 2005, RvMP, 76, 1143

Preece, R., Briggs, M. S., Mallozzi, R. S., et al. 2000, ApJS, 126, 19

Ruhl, H. 2006, in Introduction to Computational Methods in Many Particle Body Physics, ed. M. Bonitz \& D. Semkat (Paramus, NJ: Rinton Press)

Rybicki, M., \& Lightman, A. 1979, Radiative Processes in Astrophysics (San Francisco, CA: Freeman)

Sironi, L., \& Spitkovsky, A. 2009, ApJ, 707, L92

Weibel, S. 1959, PhRvL, 2, 83

Yang, T., Arons, J., \& Langdon, A. B. 1994, PhPl, 1, 3059

Yang, T., Gallant, Y., Arons, J., \& Langdon, A. B. 1993, PhFlB, 5, 3369

Yoon, P. 2007, PhPl, 14, 024504

Zhang, W., MacFadyen, A., \& Wang, P. 2009, ApJ, 692, L40 